\section{Neonatal jaundice}

Three articles on neonatal jaundice this month are essential reading. Rennie et al have summarised all the clinical guidelines for the treatment of jaundice that they found to be in use in a survey of UK neonatal units. They highlight the diversity of practice that has evolved in the face of a weak evidence base. The reappearance of kernicterus justifies a new focus on quality of practice in this area. As they point out, the rarity of this adverse outcome is such that new high quality evidence is going to be hard to come by and the way forward may be national consensus guidelines that evolve in relation to outcomes audit. Interim guidelines are proposed pending the forthcoming National Institute for Health and Clinical Excellence guidelines. Keren et al studied the ability of clinical staff to estimate the severity of jaundice in newborn infants prior to hospital discharge. The relationship between clinically assessed jaundice severity and measured bilirubin was weak. Infants assessed as not at all jaundiced did not develop jaundice that required treatment. The bottom line is that infants with visible jaundice require a bilirubin measurement if their risk of severe jaundice is to be assessed reliably. Donal Manning, lead author of the UK Surveillance Study of Severe Hyperbilirubinaemia, writes a perspective on the two studies, placing them in the context of other recent literature and stresses the need to consider all aspects of the clinical and risk management of neonatal jaundice if significant progress is to be made. See pages F314, F317 and F323

\section{Cord milking}

Hosono et al provide a secondary analysis from their RCT of umbilical cord milking. Compared with preterm infants who had their cords clamped immediately after delivery, preterm infants who got their cords milked had higher haemoglobin levels and higher blood pressure and urine output in the first days after birth. The evidence base against unphysiologically early cord clamping as a harmful intervention continues to grow. The main thing that deters delivery room staff from allowing physiological transfusion to take place seems to be a sense that urgent resuscitation of the preterm infant is required. In the small number of infants who are truly so asphyxiated as to make immediate resuscitation necessary, cord milking appears an attractive option. For the remainder of infants a comparison of milking versus allowing the blood volume augmentation to occur naturally would add valuable information. See page $\mathbf{F 3 2 8}$

\section{Premedication for intubation}

Kelleher et al repeat a survey of UK neonatal units that was first done 10 years ago regarding the use of premedication for neonatal intubation. Use has risen from $37 \%$ to $93 \%$ in the intervening period suggesting that people now widely believe that this is important. However, a big problem is the lack of evidence to point strongly towards one particular regimen, with all possible combinations having potential risks as well as benefits. Only small studies focussed on surrogate outcome measures have been performed in relevant populations. Perhaps that is why the commonest regimen is still morphine, suxamethonium and atropine. This may make the intubator feel better but the morphine won't have started working fully on the baby before the procedure starts and the suxamethonium is likely to have worn off before the trainee has got the tube in. High quality evidence may remain elusive unless large comparative studies of current regimens can take place under a waiver of consent. See page F332

\section{Always read the guarantee}

Wheeler et al report the changes in ventilator parameters delivered to newborn infants receiving volume guarantee ventilation immediately following surfactant administration. The infants responded clinically in the expected way. A brief period of airway obstruction was followed by a rapid improvement in oxygenation, with a large reduction in $\mathrm{FiO}_{2}$. Data downloaded from the ventilator showed that complete lack of tidal exchange could last up to 30 seconds and thereafter the positive inspiratory pressure required to deliver the set tidal volume remained elevated above the baseline value for 30-60 minutes. During complete obstruction the ventilator suspends volume guarantee and delivers a PIP midway between the set PEEP and the set maximum PIP. This reduction in pressure could prolong the period of obstruction and slow the establishment of improved FRC that follows treatment. If set maximum PIP was not sufficiently high above baseline, delivered tidal volume stayed below the set value for a long time after treatment. Blood gas data were not included and PEEP was not adjusted so whether the increase in delivered pressure after surfactant in this ventilation mode was the best strategy for the infant cannot be known. There are now so many newer modes of ventilation available, from different manufacturers, in use in diverse situations, that it must be few who have read all the necessary fine print to use them optimally. See page F336

\section{Sudden unexpected early postnatal collapse}

Peters et al report 5 cases where apparently well newborn infants collapsed within 12 hours of birth requiring CPR and went on to have very poor outcome. This is increasingly reported and is now the subject of a British Paediatric Surveillance Unit Study to describe the condition more fully and work towards a standardised investigation protocol (http://bpsu.inopsu.com/studies/ Neonatal_Collapse/). See page F377 